\title{
NUESTRA VULNERABILIDAD ANTE "CHOQUES" PUEDE Y DEBE SER REDUCIDA
}

\author{
Carmen Aída Lazo \\ Decana de Economía y Negocios \\ Escuela Superior de Economía y Negocios, ESEN \\ Recibido: 07/04/17 \\ Aceptado: 31/05/17
}

Los economistas llamamos «choque» (del inglés shock) a aquellos eventos que producen cambios significativos al interior de una economía, a pesar de ser externos a ella. Los choques son impredecibles, pero su impacto en el bienestar de la gente y en la estabilidad del país puede ser trascendental.

El Salvador es una nación altamente expuesta a diferentes choques, cuya frecuente ocurrencia implica importantes desafíos para las políticas públicas. Algunos de estos choques son naturales (terremotos, sequías, inundaciones), otros son provocados por la acción humana (crisis financieras, guerras civiles y desplazamientos humanos, etc.) y un tercer grupo lo constituyen las epidemias generalizadas (zika, chikunguña, H1N1).

Con el aumento de la frecuencia y el impacto de los choques a nivel mundial, en las últimas décadas se ha producido una enorme cantidad de literatura interesante dedicada a comprender la magnitud, los impactos y las implicaciones de políticas de tales choques. No obstante, los Gobiernos de muchas naciones han tardado en adoptar sistemas de identificación y reacción temprana ante tales choques, y El Salvador no es la excepción. En nuestro país, hoy en día persiste un enfoque de atención ex post a los choques, en lugar de uno ex ante.
Brevemente expondré cuatro argumentos en torno a esta temática: 1) la exposición de riesgos del país ante un choque es muy alta, 2) la frágil situación fiscal actual limita los márgenes de maniobra ante la ocurrencia de un desastre, 3 ) es necesario que el país comience a adoptar un enfoque de riesgos en su política social y económica, lo cual implicaría migrar hacia un enfoque preventivo, en lugar de uno reactivo.

\section{La magnitud de los choques}

\section{Propensión a desastres naturales}

Debido a su posición geográfica, los terremotos constituyen el riesgo más importante de desastres naturales para El Salvador. Dos de las cinco principales fuentes de actividad sísmica del país son la cadena volcánica que se distribuye a lo largo del territorio -y que forma parte del Cinturón de Fuego del Pacífico- y el movimiento de las placas tectónicas de Coco y del Caribe.

El hecho de que el país no tenga acceso al océano Atlántico y la existencia de una cadena montañosa en el norte del país actúan como una barrera natural que atenúa el impacto de los huracanes que regularmente afectan a los vecinos países de Nicaragua y Honduras. Las inundaciones son la consecuencia más común en nuestro país de los huracanes que impactan 
la región atlántica de Centroamérica.

A nivel internacional, El Salvador sobresale por presentar el mayor porcentaje de población bajo riesgo (95.4\%). Se estima que entre 1982 y 2009, el país ha tenido daños y pérdidas económicas de más de us\$6711 millones (CEPAL, 2010).

Como podemos ver en la siguiente tabla, los desastres naturales, y particularmente los terremotos, han tenido un impacto significativo en nuestro país, tanto en términos de vidas humanas como en efectos económicos adversos:

Tabla 1

Impacto de los desastres naturales en El Salvador

\begin{tabular}{llc}
\hline \multicolumn{1}{c}{ Desastre } & $\begin{array}{c}\text { Pérdidas } \\
\text { económicas } \\
\text { como \% del PIB }\end{array}$ & Muertes \\
\hline Terremoto, enero de 1982 & $2.39 \%$ & 8 \\
Terremoto, octubre de 1986 & $25.1 \%$ - 30.8\% & 1200 \\
& $1.42 \%$ & \\
Fenómeno El Niño, 1997 & $1.04 \%-3.3 \%$ & 240 \\
Tormenta tropical Mitch, 1998 & $9.13 \%$ & 844 \\
Terremoto, enero 2001 & $2.79 \%$ & 315 \\
Terremoto, febrero 2001 & $0.5 \%$ & 11 \\
Tormenta tropical Agatha, 2010 & & \\
\hline
\end{tabular}

Fuente: Lazo (2005) y CEPAL (2010).

\section{Otros choques}

En adición a los desastres naturales, El Salvador ha padecido los impactos adversos de la guerra civil, epidemias de salud y las consecuencias de los elevados niveles de violencia e inseguridad.

No cabe duda de que el país está expuesto a diversos choques que ocasionan múltiples impactos en términos de pérdida de activos privados y estatales, reducción del flujo de ingresos, pérdidas de vidas y efectos adversos en los hogares afectados. La exposición a desastres naturales como terremotos, inundaciones, sequías y otros choques puede provocar retrocesos en los avances en la reducción de la pobreza, pues existe evidencia de que estos choques tienden a afectar sobre todo a los hogares más vulnerables.
A manera de ejemplo, en la Tabla 1 se exponen los daños causados por los terremotos de 2001. De acuerdo con la CEPAL (2001), el total de daños y pérdidas causados por los terremotos ascendió a Us $\$ 1600$ millones, equivalentes al 11\% del PIв de 2001, y más de la mitad del presupuesto de ese año.

\section{Tabla 2}

Daños causados por los terremotos del 2001

\begin{tabular}{lrrr}
\hline & $\begin{array}{c}\text { 13 de } \\
\text { enero }\end{array}$ & $\begin{array}{c}\text { 13 de } \\
\text { febrero }\end{array}$ & \multicolumn{1}{c}{ Total } \\
\hline Muertes & 844 & 315 & 1159 \\
Personas heridas & 4723 & 3399 & 8122 \\
Casas destruidas & 108226 & 41302 & 149528 \\
Casas que sufrieron daños & 169632 & 15706 & 185338 \\
Edificios públicos dañados & 908 & 82 & 990 \\
Escuelas dañadas & 1366 & 200 & 1566 \\
Derrumbes & 574 & 71 & 645 \\
\hline
\end{tabular}

Fuente: Gobierno de El Salvador (2001).

¿Qué pasaría si El Salvador fuese afectado nuevamente por un choque de esta envergadura? ¿Tendría el Gobierno capacidad de respuesta?

\section{Escasos márgenes de maniobra para hacer frente a choques}

El siguiente gráfico muestra la evolución de la deuda pública total desde 1991, así como el tamaño de esta como porcentaje del рів. Cuando el país sale de la guerra civil, su nivel de deuda a PIB rondaba el $60 \%$, nivel que se reduce hasta 1998 y luego sube considerablemente, en buena medida como consecuencia de los gastos asociados a la reconstrucción de los terremotos de 2001. Se estima que los terremotos aumentaron el déficit fiscal en $1.4 \%$ en 2001 , $2 \%$ en 2002 y $1.4 \%$ en 2003 (Velasco y Rodríguez, 2004). 
Gráfico 1

Evolución de la deuda pública total (miles de millones de dólares) y deuda como porcentaje del PIB, 1991-2016

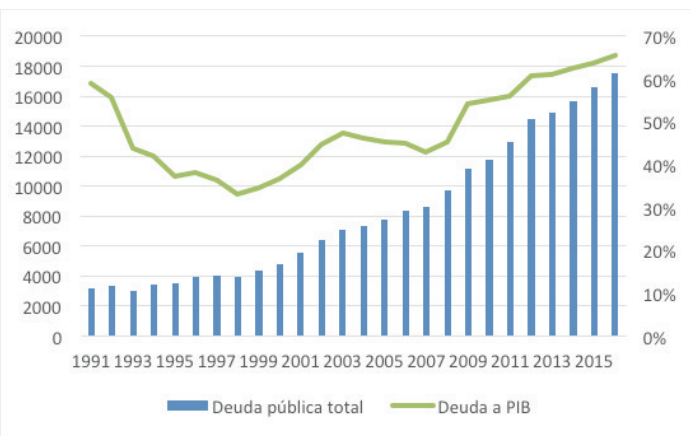

Fuente: Banco Central de Reserva.

La deuda pública total se vuelve a contener entre 2003 y 2008, para luego aumentar notablemente en 2009, atribuible en parte a la crisis financiera internacional y su impacto en la caída de la producción y el empleo. En los últimos años, y por razones no relacionadas con choques, la deuda continuó aumentando.

Como puede observar el lector, hoy en día El Salvador ha alcanzado niveles de deuda a PIB que superan los que se tenían al salir de la guerra civil. El debilitamiento de las finanzas públicas no solo se manifiesta en los elevados niveles de deuda a PIB, sino que también ha afectado el nivel de riesgo país, que es percibido por la comunidad internacional. Las agencias calificadoras han rebajado la calificación de riesgo del país en los últimos meses, y el impago temporal a la deuda previsional que se produjo en abril de 2017 ha exacerbado la preocupación por la situación fiscal del país.

En un escenario de fragilidad de las finanzas públicas como el actual, con limitado acceso a financiamiento externo, el Gobierno tendría enormes dificultades para hacer frente a un choque como el de los terremotos de 2001. De ahí la importancia que tiene el manejo macroeconómico prudente para que una nación cuente con márgenes de maniobra ante un imprevisto de semejante magnitud.

Pero hacer frente a los choques se requiere mucho más que una posición fiscal sólida. Exi- ge que los Gobiernos adopten una estrategia ex ante de identificación de riesgos y de mecanismos para financiar los costos derivados de los choques.

\section{La necesidad de prepararse para un desastre}

Ya vimos que choques como los desastres naturales pueden impactar fuertemente las condiciones económicas y sociales del país; vimos además que el país es altamente propenso a diferentes fuentes de choques y que actualmente carece de márgenes de maniobra para hacer frente de manera efectiva a un evento imprevisto de gran magnitud. Entonces, ¿qué hacer?

En primer lugar, es importante señalar que un país como El Salvador debe descartar la posibilidad de depender de la comunidad internacional de donantes ante un eventual desastre. Esto es porque la magnitud y frecuencia de desastres ha aumentado drásticamente en las últimas décadas a nivel mundial, lo que significa que existen menos recursos disponibles para países como el nuestro.

Una alternativa es acudir a la banca multilateral ante un eventual desastre para solicitar préstamos de reconstrucción. De hecho, el Banco Mundial, el BID, el BCIE cuentan con mecanismos para apoyar a los países que han sufrido diversos desastres. Sin embargo, esta estrategia ex post (en el sentido de buscar el apoyo una vez ocurrido el choque) presenta desventajas. En primer lugar, la negociación de un préstamo toma tiempo, lo cual puede reducir la efectividad de la reacción ante una crisis. En segundo lugar, el acceso a créditos de las instituciones multilaterales aumenta el nivel de endeudamiento de un país, lo cual sería particularmente complicado para nuestro país dada su frágil situación fiscal.

Una tercera posibilidad la representan los llamados «bonos catastróficos». Estos bonos funcionan de la siguiente manera: el emisor (en nuestro caso, el Gobierno de El Salvador) 
vende el bono y a cambio recibe fondos líquidos. El emisor no puede usar el dinero durante el período de cobertura, y solamente puede invertirlo en activos de bajo riesgo de corto plazo, hasta que ocurra un desastre o finalice el período de cobertura, lo que ocurriese primero. Si en el período estipulado se produce un desastre, el emisor no paga todo o parte del capital. Si no ocurre ningún desastre, el emisor debe pagar el capital y los intereses. Dicho de otra manera, los bonos catastróficos son una alternativa potencial para países vulnerables ante desastres naturales que les permiten contar con un «seguro» ante desastres para tener acceso a recursos de forma inmediata. Al igual que ocurre al pagar un seguro, esta estrategia tendría costos para el Estado, pero los bonos catastróficos evitarían el aumento súbito en los niveles de endeudamiento, además de la ventaja de inmediatez en la disponibilidad de recursos ante catástrofes.

Otra posibilidad la constituye el establecimiento de mecanismos de ahorro ex ante para hacer frente a desastres. Un ejemplo lo constituye el Fideicomiso Fondo de Desastres Naturales (Fonden), en México. Dicho Fondo, establecido por el Gobierno de México, tiene como finalidad apoyar a los diferentes entes de la administración pública y gobiernos locales en la atención y recuperación de los efectos que produzca un fenómeno natural en México.

Las estrategias anteriormente expuestas pueden y deben ser discutidas como parte de una política pública proactiva que busque identificar estrategias en antelación a la ocurrencia de desastres. En adición a la búsqueda de recursos de manera preventiva, el Gobierno puede redoblar esfuerzos por asegurar la infraestructura pública (lo que reduciría la necesidad de buscar recursos para la reconstrucción de la misma) y establecer sistemas de información que permitan mapear la exposición a choques de la población.

Este enfoque de identificación de riesgos permitiría que la política social no se concentre exclusivamente en atender a quienes ya están en condición de pobreza, sino también en quienes pueden caer en ella por su alta exposición a diferentes choques. La información estadística proveniente de diferentes fuentes podría ser consolidada por la Dirección General de Estadísticas y Censos (Digestyc) u otra instancia que estuviese vinculada con el mapa de riesgos, y así facilitar la identificación temprana de poblaciones vulnerables que están siendo impactadas por choques y las medidas inmediatas para atenuar los impactos de mediano plazo.

En definitiva, lo menos que puede hacer una nación altamente expuesta a choques es prepararse para estos y mitigar así consecuencias adversas en su estabilidad económica y en el bienestar de su población.

\section{FUENTES CONSULTADAS}

Comisión Económica para América Latina y el Caribe-CEPAL Evaluación de daños y pérdidas en El Salvador ocasionados por la tormenta tropical Agatha. México: Naciones Unidas.

Comisión Económica para América Latina y el Caribe-CEPAL (2001). El terremoto del 13 de enero de 2001 en El Salvador. Impacto socioeconómico y ambiental. México: Naciones Unidas.

Escuela Superior de Economía y Negocios-EsEN (2002). Mecanismos financieros para el manejo de los riesgos provenientes de los desastres naturales. San Salvador: ESEN.

Gobierno de El Salvador. Unidos por El Salvador: Plan de recuperación de los daños causados por los terremotos del 13 de enero y 13 de febrero de 2001. Reunión Grupo Consultivo, coordinada por el Banco Interamericano de Desarrollo, Madrid, España, marzo, 2001.

Lazo, C. A. (2005). Preparing for Disasters: Financing Earthquake Reconstruction in El Salvador (mimeo). Cambridge: Harvard University.

Velasco, A. \& Rodríguez, C. (2004). El Salvador: Fiscal Performance, Prospects and Policy Options. Inédito.

World Bank (2001). «Managing Catastrophic Disaster Risks Using Alternative Risk Financing and Pooled Insurance Structures». Technical Paper (495), May 2001. 